\title{
Railway modelling for power quality analysis
}

\author{
M. Chymera, A. C. Renfrew \& M. Barnes \\ University of Manchester, UK
}

\begin{abstract}
Power quality is increasingly important in the consideration of railway systems. Poor power quality affects the performance of trains and introduces problems for local distribution networks.

This paper looks at improving modelling techniques to assess the power quality of a railway system. Modelling techniques used to simulate electrified railways are adapted to simulate power quality aspects with improved computational efficiency. The model developed is used to assess the voltage regulation and harmonic distortion of a rail system, and harmonic distortion on the local distribution network.
\end{abstract}

Keywords: power quality, harmonics, electric traction, modelling.

\section{Introduction}

Power quality has become an increasing concern in railway systems. Poor power quality affects the performance, reliability of the railway system as well as having an effect on equipment attached to the local distribution network. The analysis of power quality on a rail system is essential, to enable the analysis of train performance and to assess the effects of a rail system on the adjacent distribution network.

Railway systems are electrically complex. The loads, trains, are constantly moving and their electrical behaviour is constantly changing. Modelling is an ideal tool to analyse the power quality of such a complex system. An ideal model would be accurate yet computationally efficient.

\section{Power quality}

Power qQuality has to be considered from two perspectives, the railway system and the local distribution network to which it is attached. Poor power quality on 
the railway system affects performance, limits capacity and may introduce a need for additional maintenance. Distribution network operators stipulate power quality standards. Railway systems are required to meet these standards.

Power quality is divided into categories [1]. Railway systems typically suffer from poor voltage regulation and harmonic distortion. Voltage regulation affects the performance of traction units and imposes a capacity limit on the system. Harmonic distortion can cause damage to traction units, particularly motors [2].

Rail systems can cause harmonic distortion and voltage unbalance on the local distribution network. The harmonic distortion is caused by the non-linear currents drawn by the railway system and in DC systems by rectifying substations. Voltage unbalance is present in AC systems, where the rail system draws large currents from a single phase of the supply.

\section{Modelling railway power quality}

Modelling is required to assess the power quality of a rail system and the effect of the rail system on the local distribution network. Analysis is essential for assessing existing systems to establish the need for power quality compensation, or improvements to systems, and for the analysis of new systems or the effect of major improvements to an existing system.

Various modelling techniques exist for the analysis of railway electrical behaviour. Many models are developed to simulate train movement. Some models have been produced to analyse power quality, however these models tend to focus on single aspects of power quality, such as harmonic analysis [3] and voltage regulation [4]. It would be desirable and more effective to simulate these aspects of power quality together.

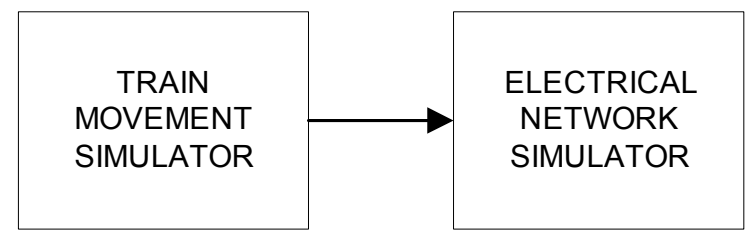

Figure 1: Rail system model.

Figure 1 shows the model developed to simulate power quality in a rail system. The train movement simulator uses input data to calculate train positions and electrical behaviour. The electrical network simulator uses the positions to calculate the line impedances, and load currents.

\section{Power quality analysis}

Power quality can be analysed from voltage and current waveforms. To produce voltage and current waveforms the rail system has to be analysed using differential equations. This requires short time steps and results in long computation times when performed as a time stepping transient analysis. 
Assessing some of the relevant power quality aspects individually in the frequency domain may result in more efficient simulation.

\subsection{Voltage regulation}

Voltage regulation can be analysed by using steady-state analysis. The rail system is modelled as an electrical network and the network is analysed at each time step. Nodal voltage analysis provides an ideal tool for modelling voltage regulation.

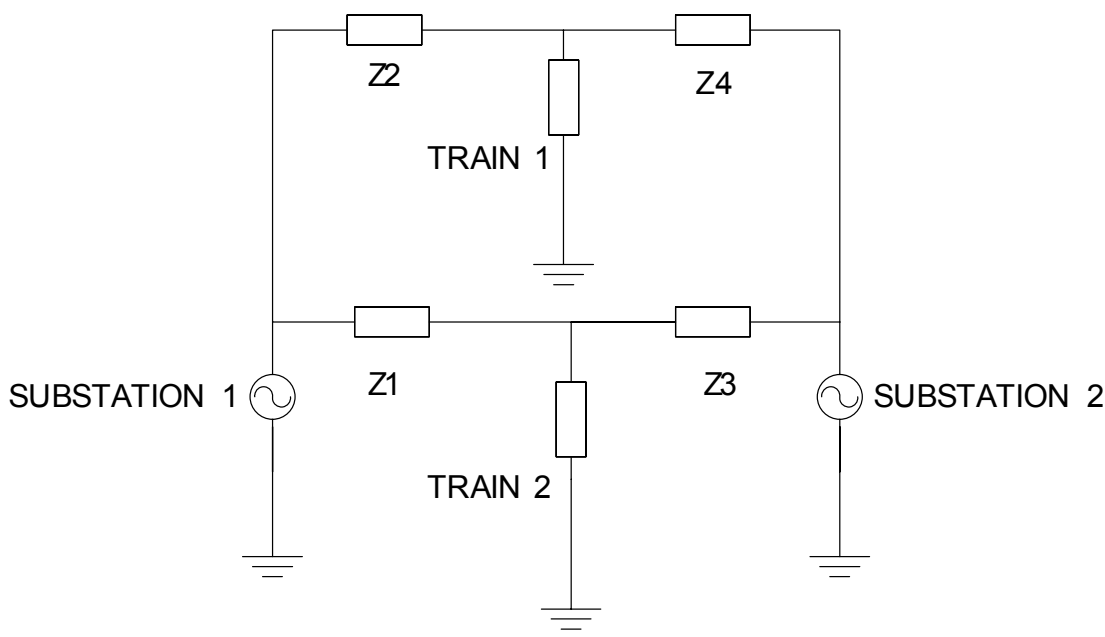

Figure 2: Electrical representation of a simple railway system.

Nodal voltage analysis uses matrices to represent the rail system. A simple rail system, Figure 2 can be represented using eqn (1). The line impedances are calculated from the train positions.

$$
\left(\begin{array}{cc}
\frac{1}{\mathrm{Z}_{1}} & \frac{1}{\mathrm{Z}_{3}} \\
\frac{1}{\mathrm{Z}_{2}} & \frac{1}{\mathrm{Z}_{4}}
\end{array}\right)\left(\begin{array}{c}
\mathrm{V}_{\text {substation1 }} \\
\mathrm{V}_{\text {substation2 }}
\end{array}\right)=\left(\begin{array}{cc}
\frac{1}{\mathrm{Z}_{1}}+\frac{1}{\mathrm{Z}_{3}} & 0 \\
0 & \frac{1}{\mathrm{Z}_{2}}+\frac{1}{\mathrm{Z}_{4}}
\end{array}\right)\left(\begin{array}{c}
\mathrm{V}_{2} \\
\mathrm{~V}_{3}
\end{array}\right)+\left(\begin{array}{c}
\mathrm{I}_{\mathrm{T} 1} \\
\mathrm{I}_{\mathrm{T} 2}
\end{array}\right)
$$

\subsection{Harmonic distortion}

Conducting frequency domain analysis and performing network analysis for each harmonic frequency offers a more efficient method of analysing waveform distortion, if superposition can be assumed. The model of each train must consider the harmonics currents drawn. This means each load produces a table of harmonics including a frequency and a magnitude. 
Harmonic distortion is considered within the railway system and on the local distribution network. The harmonic distribution is simulated in 5 stages:

1. Model of the substation is configured for the railway side.

2. The magnitudes of harmonic currents drawn by the train are calculated

3. Network analysis is performed on all the frequencies present.

4. The external models of the substations are constructed.

5. Network analysis of all frequencies is performed for the distribution network.

\subsubsection{Substation model - railway side}

For an AC system the substation is modelled as an AC source, to represent the fundamental. If there is significant harmonic distortion on the distribution network, additional voltage sources are included in this model. The magnitude of the sources depends on the behaviour of the distribution network. The distribution network model is simplified to a static model and hence the magnitudes of the voltage sources are predetermined and stored in a table.

For a DC system, where the substation contains a transformer and a rectifier, the substation is modelled as a set of voltage sources at various frequencies. The magnitude of each voltage source is dependent on the total current drawn from the substation. The current drawn is determined when the analysis of the DC voltage is considered, after the initial network analysis is complete, the substation ripple frequency magnitudes are determined.

\subsubsection{Train harmonic model}

Modern trains employ power electronic drives which draw harmonic currents. The harmonics drawn depend on the type of drive system and the state of the train (acceleration and speed). Analysing the train, the harmonics drawn can be calculated. Equations can be established to determine harmonic currents drawn by the train. The train model must also consider the attenuation of harmonic currents by filters.

\subsubsection{Railway side harmonic analysis}

Network analysis, employing nodal voltage analysis is used to simulate the harmonics in the railway system. The equation used for the voltage regulation analysis, eqn (1) is used to perform the analysis of each harmonic. The impedances per unit length are calculated for each frequency using eqn (2). The line impedances are calculated from the train positions and the impedance per unit length.

$$
Z=\sqrt{R^{2}+(2 \pi f L)^{2}}
$$

\subsubsection{Substation model - grid side}

The currents drawn by the rail system from each substation at various frequencies have been determined; the substation model is used to determine the currents drawn from the grid. For an AC system the harmonics drawn from the substation are the same as those drawn by the railway system. For DC systems 
the harmonics drawn are the harmonics of the system plus the harmonics drawn by the rectifier.

\subsubsection{Distribution network analysis}

The distribution network is modelled, with the rail system as a load, Figure 3. A matrix is constructed to represent the distribution network impedances. Using harmonic data from the sources, and transmission line impedances the network is analysed. The analysis can be performed for test systems [5] to perform general harmonic analysis of a rail system.

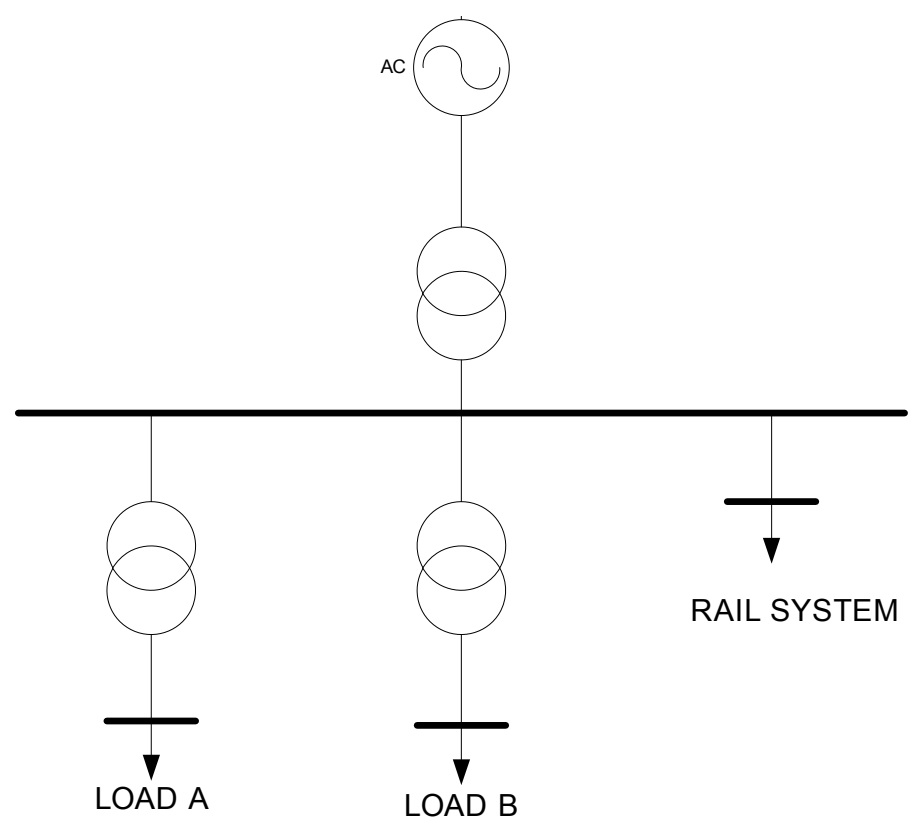

Figure 3: Simple distribution network.

\section{Modelling the Blackpool Tramway}

The modelling techniques were used to simulate the Blackpool Tramway, a $550 \mathrm{~V}$ DC tram system.

\subsection{Tram model}

Blackpool tram system employs thyristor control trams. The chopper operates between $40 \mathrm{~Hz}$ and $800 \mathrm{~Hz}$. The Blackpool Tram's use a filter to attenuate ripple, the filter characteristics are shown in Figure 4. The filter significantly attenuates ripple, and hence the ripple currents drawn by the tram are considered negligible.

The substation on the Blackpool use 12-pulse rectifiers; a 12 pulse rectifier frequency domain model is represented in Table 1. 


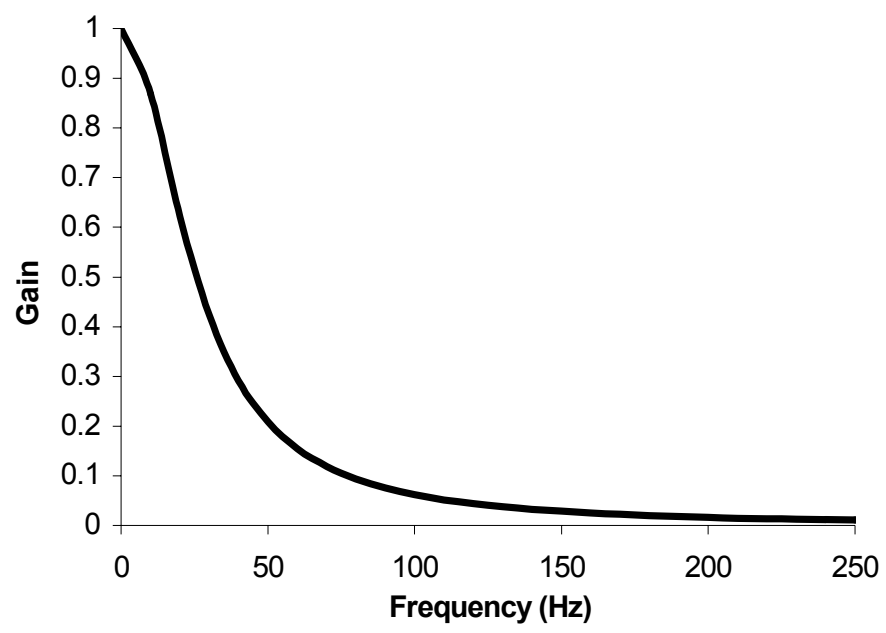

Figure 4: Blackpool Tram filter characteristic.

Table 1: Frequency domain model for substation (railway side).

\begin{tabular}{|l|l|l|}
\hline Frequency/Hz & 600 & 1200 \\
\hline Magnitude/A & $0.014 \mathrm{I}$ & $0.010 \mathrm{I}$ \\
\hline
\end{tabular}

Table 2: Frequency domain model of substation (distribution network side).

\begin{tabular}{|l|l|l|l|l|l|l|}
\hline Frequency/Hz & 550 & 650 & 1150 & 1250 & 1750 & 1850 \\
\hline Magnitude/A & $0.0644 \mathrm{I}$ & $0.0329 \mathrm{I}$ & $0.0110 \mathrm{I}$ & $0.0108 \mathrm{I}$ & $0.0053 \mathrm{I}$ & $0.0042 \mathrm{I}$ \\
\hline
\end{tabular}

The external model of the railway hence considers a linear load on a 12-pulse rectifier. The harmonic currents drawn by the rail system are dependent on the current. Table 2 represents the harmonic currents as a function of current.

\section{Results}

\subsection{Voltage regulation}

Figure 5 shows the voltage regulation curve for a tram on the Blackpool Tramway as the tram starts from rest and accelerates to $30 \mathrm{mph}$, the tram then drives for 5 minutes before a short period of coasting and braking. The curve shows a voltage minimum of $537 \mathrm{~V}$.

\subsection{Harmonic analysis}

The simulation results demonstrate a constant DC voltage ripple of $1.7 \%$ within the tram system. The total harmonic current distortion at the substation is $7.6 \%$. The Simulation results showed a negligible effect of the tram system on the Total Harmonic Voltage distortion of the local distribution network. 


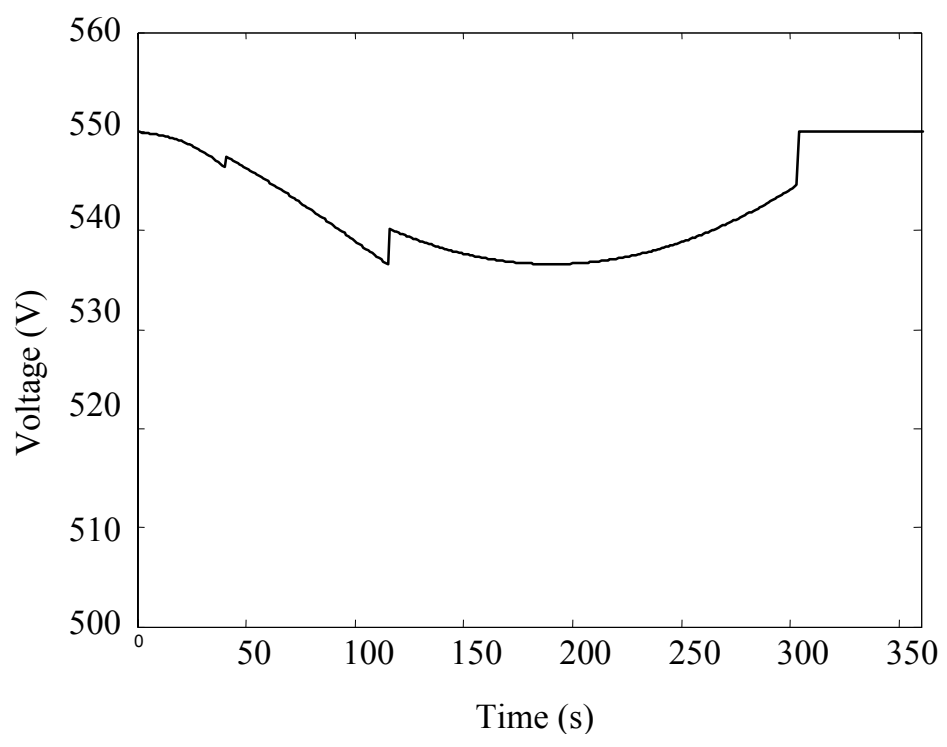

Figure 5: Voltage regulation on the Blackpool Tramway.

\section{Discussion}

The simulation results of the Blackpool system showed that the Blackpool tramway has reasonable voltage regulation at its current usage levels. The harmonic analysis showed a small ripple within the system of $1.7 \%$, this figure is small enough not to cause any interference concerns. The harmonic analysis on the external systems showed that there is significant harmonic current distortion; however this does not propagate onto the distribution network due to the small relative size of the system.

\section{Conclusion}

The modelling techniques described provide an effective way of simulating rail system power quality. Results show the voltage regulation and harmonic distortion on the railway network and on the local distribution network. The tools can be used in the design or modification of railway schemes. The voltage regulation output is an indicator of the railways potential performance. The harmonic analysis allows the identification of any potential interference problems, particularly electromagnetic interference with signalling circuits. The harmonic analysis on the distribution network can be used to check if the grid code standards are met, and to assess the requirements for compensation.

The next stages of the work require verification of the model. Experimental results are required to assess the accuracy of the model. 


\section{Acknowledgements}

The Authors would like to thank the Engineering Physical Science Research Council and HILTech Developments Ltd, who are funding the research. Thanks also to Blackpool Transportation and its Chief Electrical Engineer Peter Brown, who have provided data on the Blackpool Tramway, used to construct the models described.

\section{References}

[1] Schlabbach, J., Blume, D. \& Stephanblome, T., Voltage Quality in Electrical Power Systems. London: IEE, 2001.

[2] Lee, C.Y., \&Lee, W.J., Effects of nonsinusoidal voltage on the operation performance of a three-phase induction motor. IEEE Transactions on Energy Conversion, 14, pp. 193-201, 1999.

[3] Chang, G.W., Lin, H.W., \& Chen, S.K., Modeling characteristics of harmonic currents generated by high-speed railway traction drive converters. Power Delivery, IEEE Transactions on, 19, pp. 766-773, 2004.

[4] Hill, R.J., \& Cevik, I.H., On-line simulation of voltage regulation in autotransformer-fed AC electric railroad traction networks. Vehicular Technology, IEEE Transactions on, 42, pp. 365-372, 1993.

[5] Abu-Hashim, R., Burch, R., Chang, G., Grady, M., Gunther, E. , Halpin, M., Harziadonin, C., Liu, Y., Marz, M., Ortmeyer, T., Rajagopalan, V., Ranade, S., Ribeiro, P., Sim, T., \& Xu, W., Test systems for harmonics modeling and simulation IEEE Transactions on Power Delivery, 14, pp. 579-87, 1999. 